\title{
Marrow fat content and composition in $\beta$-Thalassemia: a study using 1H-MRS
}

\begin{abstract}
Background: $\beta$-thalassemia is a genetic disease that causes abnormal production of red blood cells (ineffective erythropoiesis, IE). IE is a condition known to change bone marrow composition. Purpose: To evaluate the effect of IE on the marrow fat content and fat unsaturation levels in the proximal femur using 1 H-MRS. Study Type: Prospective. Subjects: Twenty-three subjects were included in this study, seven control and $16 \beta$-thalassemia subjects. Field Strength/Sequence: 3.0T; stimulated echo acquisition Mode (STEAM); magnetic resonance spectroscopy (MRS) sequence. Assessment: Multiecho MRS scans were performed in four regions of the proximal left femur of each subject, that is, diaphysis, femoral neck, femoral head, and greater trochanter. The examined regions were grouped into red (diaphysis and femoral neck) and yellow marrow regions (femoral head and greater trochanter). Statistical Tests: The Jonckheere-Terpstra test was used to evaluate the impact of increasing disease severity on bone marrow fat fraction (BMFF), marrow conversion index, and fat unsaturation index (UI). Pairwise comparison analysis was performed when a significant trend $(\mathrm{P}<0.05)$ was found. K-means clustering analysis was used to examine the clusters observed when BMFF in the red and yellow regions were studied (diaphysis against greater trochanter). Results: BMFF showed a significant decreasing trend with increasing disease severity in both red $(\mathrm{TJT}=109.00, \mathrm{z}=-4.414, \mathrm{P}<0.05)$ and yellow marrow regions $(\mathrm{TJT}=108.00, \mathrm{z}=-$ 4.438, $\mathrm{P}<0.05$ ). The opposite trend was observed in UI in both bone marrow regions (red marrow: $\mathrm{TJT}=180.5, \mathrm{z}=3.515, \mathrm{P}<0.05$; yellow marrow: $\mathrm{TJT}=155.0, \mathrm{z}=2.282, \mathrm{P}=0.05$ ). Three distinct forms of marrow adipogenesis were found when plotting BMFF diaphysis against BMFF greater trochanter: 1) normal (centroid: 80.4\%, 66.6\%), 2) partial disruption (centroid: $51.1 \%, 16.6 \%$ ), and 3 ) total disruption (centroid: $2.6 \%, 1.6 \%$ ). Data Conclusion: $\beta$ thalassemia is associated with decreased marrow fat, and increased marrow fat unsaturation level. Level of Evidence: 2 Technical Efficacy Stage: 3.
\end{abstract}

Keyword: MRS; Bone marrow fat; Hematopoiesis; Ineffective erythropoiesis; Marrow adipose tissue (MAT); $\beta$-thalassemia 